\title{
Premedication with atropine+ketamine vs atropine+ketamine+/-propofol for less invasive surfactant administration: a prospective comparative
} study

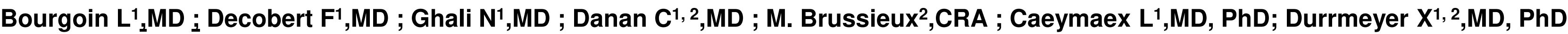 \\ 1: Neonatal Intensive Care Unit, CHI Créteil, Créteil, Université Paris Est Créteil, France \\ 2: Clinical Research Centre, $\mathrm{CHI}$ Créteil, Créteil, Université Paris Est Créteil, France
}

Contacts : laura.bourgoin@chicreteil.fr; ; xavier.durrmeyer@chicreteil.fr

\section{Background}

Less invasive surfactant administration (LISA) is associated with decreased mechanical ventilation and reduced bronchopulmonary dysplasia in premature infants ${ }^{1}$. However, analgosedation was rarely used in controlled trials ${ }^{1}$ in spite of the known pain and discomfort induced by the laryngoscopy this technique requires.

When we implemented this technique in our unit in 2015, we used routine premedication with atropine+ketamine, which resulted in a relatively high rate of subsequent intubation ${ }^{2}$.

In 2016, we modified our premedication protocol by limiting the dose of ketamine and adding propofol if required.

We hypothesized that this new protocol would reduce the intubation rate.

\section{Objective}

To compare the efficacy (pain control) and the tolerance (intubation rate) of a premedication using atropine + ketamine +/- propofol vs atropine + ketamine prior to a LISA procedure.

\section{Design \& Setting}

\section{Methods}

Prospective study in a single level III center's Neonatal Intensive Care Unit comparing 2 periods with different premedication protocols.

\section{Patients}

As per local protocol, infants were eligible for a LISA procedure if they met the following criteria:

- Gestational age $>25$ weeks +6 days and $<37$ weeks

Receiving non-invasive ventilation

- Diagnosis of neonatal RDS requiring surfactant administration based on clinical signs and chest Xray

$\mathrm{FiO}_{2}$ thresholds for surfactant administration were:

- $\geq 0.25$ for infants born $<28$ weeks

$\geq 0.30$ for infants born $\geq 28$ weeks

Contra-indication to a LISA procedure were:

Pneumothorax

Hemodynamic instability

Frequent apnea and bradycardia

Hypercapnia > $65 \mathrm{~mm} \mathrm{Hg}$

\section{Intervention - Local protocol}

From March 2015 to November 2016 (KETA period) we used atropine+ketamine titrated by $0.5 \mathrm{mg} / \mathrm{kg}$ increments. From November 2016 to March 2018 (KETAPRO period), we used atropine + a single dose of ketamine with an optional single dose of propofol if satisfactory sedation was not achieved. Drugs' doses used are summarized in Table 1.

\begin{tabular}{|c|c|c|}
\hline \multicolumn{3}{|c|}{ Table 1 : Drugs' doses } \\
\hline Drugs & $\begin{array}{c}\text { KETA } \\
\text { (March 15-Nov 16) }\end{array}$ & $\begin{array}{c}\text { KETAPRO } \\
\text { (Nov } 16-\text { March 18) }\end{array}$ \\
\hline $\begin{array}{l}\text { Atropine } \\
(\mu \mathrm{g} / \mathrm{kg})\end{array}$ & 20 & 20 \\
\hline $\begin{array}{l}\text { Ketamin } \\
\mathrm{e}(\mathrm{mg} / \mathrm{kg})\end{array}$ & $\begin{array}{l}\text { Increments of } 0.5 \\
\text { Maximal dose }: 3\end{array}$ & $\begin{array}{c}\text { Unique dose } \\
0.5 \text { if } G A<30 \text { weeks } \\
1 \text { if } G A \geq 30 \text { weeks }\end{array}$ \\
\hline $\begin{array}{l}\text { Propofol } \\
(\mathrm{mg} / \mathrm{kg})\end{array}$ & - & $\begin{array}{c}\text { Unique dose } \\
\text { only if adequate sedation } \\
\text { was not achieved } 5 \text { min } \\
\text { after ketamine: } \\
0.5 \text { if } \mathrm{GA}<30 \text { weeks } \\
1 \text { if } \mathrm{GA} \geq 30 \text { weeks }\end{array}$ \\
\hline
\end{tabular}

Results

From December 2016 to March 2018, 18 infants were included in the study and were compared to 40 infants from the previous period. Their baseline characteristics are summarised in Table 2.

\begin{tabular}{lccc}
\hline \multicolumn{4}{c}{ Table 2: Baseline characteristics expressed as means (SD) } \\
\hline & $\begin{array}{c}\text { KETA } \\
\text { KETAPRO }\end{array}$ & $\begin{array}{c}\text { Karch15-Nov16) } \\
\text { (Nov16- March18) }\end{array}$ & $\mathrm{p}$ \\
$\mathrm{GA}$ at birth, wks & $30.1(2.3)$ & $30.2(2.9)$ & 0.94 \\
Birth weight, g & $1384(561)$ & $1300(499)$ & 0.59 \\
$\mathrm{FiO}_{2}$ at LISA, \% & $35(12)$ & $39(9)$ & 0.20 \\
\hline
\end{tabular}

The description and tolerance of the LISA procedure in the 2 periods are summarised in Table 3. During the KETAPRO period 15/18 infants received propofol and the doses of ketamine used were significantly reduced as compared to the KETA period.

\begin{tabular}{|c|c|c|c|}
\hline \multicolumn{4}{|c|}{ Table 3: Description of the LISA procedure } \\
\hline & $\begin{array}{c}\text { KETA } \\
\text { (March15-Nov16) } \\
n=40\end{array}$ & $\begin{array}{c}\text { KETAPRO } \\
\text { (Nov16- March18) } \\
n=18\end{array}$ & $\mathrm{p}$ \\
\hline $\begin{array}{l}\text { Median [IQR] } \\
\text { ketamine dose, } \\
\mathrm{mg} / \mathrm{kg}\end{array}$ & $1.5[1-2.5]$ & $1[0.5-1]$ & $<10^{-4}$ \\
\hline $\begin{array}{l}\text { Median [IQR] } \\
\text { propofol dose, } \mathrm{mg} / \mathrm{kg}\end{array}$ & (1 infant: 2) & $0.75[0.5-1]$ & - \\
\hline Apnea, n (\%) & $19(48)$ & $9(50)$ & 0.78 \\
\hline $\begin{array}{l}\text { Intubation before } \\
\text { LISA, } n(\%)\end{array}$ & $7(18)$ & $3(18)$ & 1.00 \\
\hline $\begin{array}{l}\text { Median [IQR] } \\
\text { number of } \\
\text { laryngoscopy }\end{array}$ & $2[1-4]$ & 1 [1-2] & 0.42 \\
\hline $\begin{array}{l}\text { Desaturation < 85\%, } \\
\mathrm{n}(\%)\end{array}$ & $25(63)$ & $14(78)$ & 0.20 \\
\hline $\begin{array}{l}\text { Median [IQR] lowest } \\
\mathrm{SpO}_{2} \text { value, \% }\end{array}$ & 59 [20-92] & $43[23-56]$ & 0.06 \\
\hline $\begin{array}{l}\text { Intubation < } 72 \mathrm{~h}, \mathrm{n} \\
(\%)\end{array}$ & $18(45)$ & $8(44)$ & 1.00 \\
\hline Pain assessment & $n=30$ & $n=16$ & \\
\hline $\begin{array}{l}\text { Median [IQR] } \\
\text { Faceless Acute } \\
\text { Neonatal Pain Score } \\
\text { (FANS) }\end{array}$ & $2[2-4]$ & $2[2-4]$ & 1.00 \\
\hline FANS < 4, n (\%) & $18(60)$ & $10(63)$ & 1.00 \\
\hline
\end{tabular}

\section{Discussion}

The optional addition of propofol and the limitation of ketamine's dose was not associated with a decrease in the immediate or delayed intubation rate. We observed a trend towards lower $\mathrm{SpO}_{2}$ nadirs in the KETAPRO period.

Propofol should probably be used as a single agent with a unique dose in order to improve its respiratory tolerance, as suggested in other studies ${ }^{3-5}$.

\section{Conclusion}

Obtaining adequate and safe sedation during LISA in challenging. Combining ketamine and propofol had no advantage over ketamine alone in our population.

1: Aldana-Aguirre et al Arch Dis Child Fetal Neonatal Ed. 2017 102(1):F17-F23. 2: Bourgoin L et al. Acta Paediatr. 2018;107(7):1184-1190 3: Dekker J et al. Arch Dis Child Fetal Neonatal Ed. 2018 Aug 1. pii: fetalneonatal-2018-315015. 4: Descamps CS et al. Arch Dis Child Fetal Neonatal Ed. 2017 Sep;102(5):F465 5: Dekker J et al. Neonatology. 2016;109(4):308-13. 